\title{
Eugenio Filloy Yagüe: un breve recuento de vida y obra ${ }^{1}$
}

\author{
29 de noviembre de 1942 - 23 de marzo de 2020
}

\author{
Armando Solares ${ }^{2}$ \\ Luis Puig ${ }^{3}$ \\ Teresa Rojano 4
}

En 1939, Soledad Yagüe Herranz de Filloy y Enrique Filloy Méndez, el matrimonio Filloy Yagüe, cruzan el Atlántico como consecuencia de la derrota de la República Española a manos de los sublevados encabezados por el general Francisco Franco. Instalados en San Pedro de Macorís, República Dominicana, nace en 1942 el tercero de sus hijos, Eugenio. En agosto de 1945, la familia de Eugenio llega a México en el buque Emancipación. Aquí encontrarán su tierra.

Eugenio estudia en el Instituto Luis Vives y, posteriormente, se forma como matemático en la Facultad

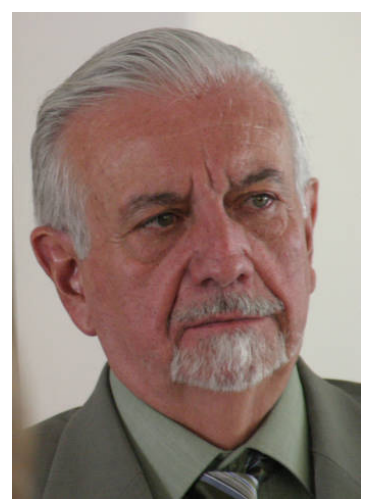
de Ciencias de la Universidad Nacional Autónoma de México, donde se licencia en 1965 con el trabajo "Representaciones de grupos finitos". Inmediatamente, ingresa a trabajar como profesor adjunto de la Escuela Superior de Física y Matemáticas del Instituto Politécnico Nacional y, en paralelo, estudia la maestría en el Departamento de

${ }^{1}$ Esta semblanza está basada en anteriores homenajes al trabajo y vida de Eugenio Filloy, los cuales fueron presentados por Luis Puig y Teresa Rojano tanto en el Cinvestav como en la Universidad Pedagógica Nacional, en México.

${ }^{2}$ Departamento de Matemática Educativa, Cinvestav-IPN, asolares@cinvestav.mx, México

${ }^{3}$ Departamento de Matemática Educativa, Cinvestav-IPN, trojano@cinvestav.mx, México

${ }^{4}$ Departamento de Didàctica de la Matemàtica, Universitat de València Estudi, Generalluis.puig@uv.es, España 
Matemáticas del Centro de Investigación y Estudios Avanzados (CINVESTAV), graduándose en 1966. A raíz de la matanza del 2 de octubre de 1968, Eugenio cruza la frontera mexicana y se traslada a la Universidad de Chicago en el año 1969. Con rapidez y calidad ambas sobresalientes, en 1970 obtiene el doctorado en matemáticas con la tesis "Pointwise characterization of ideals of differentiable functions", bajo la dirección de Raghavan Narasimhan, que se publicaría como artículo en Invention Maths de Springer (Filloy, 1971).

En enero de 1971, Eugenio Filloy regresa a México con una vigorosa trayectoria como matemático y se integra como Investigador Adjunto al Departamento de Matemáticas del CINVESTAV. En su retorno, se introduce al trabajo en el campo educativo por medio de un proyecto que tendrá un enorme impacto en el sistema educativo mexicano y, también, en el desarrollo del mismo, Eugenio, como científico e impulsor de proyectos participa en la coautoría de los libros de texto de matemáticas de nivel primaria, junto con Carlos Imaz, Samuel Feder, Samuel Gitler, Luis Gorostiza y Juan José Rivaud, publicados en ediciones sucesivas, entre 1972 y 1978, por la Secretaría de Educación Pública. De hecho, este proyecto fue el germen de la creación, en 1975, de la entonces Sección de Matemática Educativa del CINVESTAV, a la cual Eugenio se incorporó ya como Investigador Titular.

En el primer frente de la constitución del área de la Matemática Educativa en México, Eugenio orienta su desarrollo de manera que las "Matemáticas" son el substantivo en la investigación. En 1981, la Sección de Matemática Educativa abre el programa de doctorado y, por iniciativa de Eugenio, la sección se reestructura en áreas que permiten atender tanto a las problemáticas de los distintos niveles escolares (básico, medio superior y superior) como a los dominios específicos de investigación, como la cognición matemática y el desarrollo de materiales didácticos utilizando tecnologías.

En 1983, Eugenio pone en marcha uno de los programas más ambiciosos de formación de maestros que han existido: el Programa Nacional de Formación y Actualización de Profesores de Matemáticas (PNFAPM), que hasta ya entrados los años 2000 incorpora a docentes de matemáticas a programas de especialidad y maestría impartidos en veinte universidades estatales y diez tecnológicos regionales. Para acompañar el desarrollo de este programa, Filloy diseña la realización de simposios internacionales y convenios de investigación con el King's College y el Instituto de Educación de la Universidad de Londres del Reino Unido, y con las Universidades de Valencia y de La Laguna en España, en el marco de los cuales se realizan tesis doctorales y de maestría, así como proyectos de investigación 
conjuntos. Puede decirse que una de las consecuencias del PNFAPM es la consolidación de la Sección del Departamento de Matemática Educativa, en 1992.

Con todo este trabajo, Eugenio Filloy está en la posición de generar una perspectiva teórica original para la Matemática Educativa, con componentes propios de su visión de hacer ciencia: el uso del análisis histórico de las ideas matemáticas, las entrevistas clínicas con enseñanza, la observación en un sistema de enseñanza controlada, el carácter local y de modelo de lo que usamos para el análisis de lo observado, la consideración de las situaciones de enseñanza y aprendizaje de las matemáticas como situaciones de comunicación y producción de sentido, y, por tanto, susceptibles de ser abordadas desde una perspectiva semiótica.

Respecto al uso de la historia, Eugenio propone realizar una lectura de textos históricos de las matemáticas tomándolos como cogniciones petrificadas, de forma que puedan servir para proponer hipótesis sobre las cogniciones de los alumnos cuando están siendo enseñados, y, a su vez, leer las producciones de los alumnos de forma que proporcionen hipótesis con las que leer los textos históricos. Las dificultades y fenómenos que encuentran en torno a la operación con la incógnita (Rojano, 1985), en el momento en que los estudiantes son introducidos al pensamiento algebraico, son el ejemplo paradigmático de esta ida y vuelta entre los textos históricos y los textos producidos en un modelo de enseñanza.

Por otra parte, Filloy plantea realizar entrevistas con intervención de enseñanza como un instrumento metodológico adecuado para estudiar los fenómenos que se producen cuando se enseñan matemáticas en los sistemas escolares. Para ello, es necesaria la creación de sistemas de enseñanza controlada que hagan posible tener una descripción de los alumnos que van a ser entrevistados, desde el punto de vista de lo que se quiere investigar. Es así que Eugenio construye el Centro Escolar Hermanos Revueltas, una propuesta escolar alternativa y estrechamente vinculada a la investigación educativa que estuvo en funcionamiento hasta hace unos pocos años.

Eugenio Filloy introduce la idea de Modelos Teóricos Locales para hacer explícitos el conjunto de supuestos que permiten organizar y analizar las observaciones sobre los fenómenos investigados. Desde la perspectiva de Filloy, estos supuestos se hacen explícitos sin pretender que sean la única manera de organizar y analizar los fenómenos -es decir, sin afirmar que constituyan una teoría y, por tanto, tener que rechazar otros supuestos. Estos modelos son, además, "locales" en el sentido de que permiten dar cuenta precisamente de lo observado en cada ocasión específica y concreta. Eugenio sintetiza esta perspectiva teórica en el libro Educational Algebra. A Theoretical and Empirical Approach (Filloy, Rojano y Puig, 2008). 
Desde esta perspectiva teórica, Filloy propone y deja planteados numerosos temas y problemáticas de investigación que aún están en desarrollo, como los fenómenos de producción de sentido y significado de los textos matemáticos, los algebraicos, en concreto, (Filloy, Rojano y Solares, 2010) y la noción de intertextualidad (Rojano, Filloy y Puig, 2014).

A lo largo de su trayectoria de trabajo, Eugenio Filloy, apoya e impulsa la creación y consolidación de muchas de las más importantes organizaciones y espacios de difusión internacionales dedicadas a la investigación en nuestra área, como la CIEAEM, el PME, y el ICME.

Su trayectoria académica queda registrada en 456 publicaciones nacionales e internacionales que a la fecha suman cerca de mil citas. Formó a cerca de 100 investigadores, a través de programas de maestría y doctorado. Investigador Emérito del CINVESTAV, miembro del Sistema Nacional de Investigadores Nivel III.

Científico, erudito, visionario, amigo. Su obra nos acompaña y queda en nuestras memorias.

\section{REFERENCIAS}

Filloy, E. (1971). Pointwise characterization of ideals of differentiable functions. Inventiones mathematicae, 13, 143-168. https://doi.org/10.1007/BF01390098

Filloy, E., Rojano, T. y Puig, L. (2008). Educational Algebra. A Theoretical and Empirical Approach. Springer.

Filloy, E., Rojano, T. y Solares, A. (2010). Problems Dealing With Unknown Quantities and Two Different Levels of Representing Unknowns. Journal for Research in Mathematics Education, 41(1), 55-80. www.jstor.org/stable/40539364

Rojano, T. (1985). De la artitmética al álgebra: un estudio clínico con niños de 12 a 13 años de edad. (Tesis doctoral), Centro de Investigación y Estudios Avanzados, México.

Rojano, T., Filloy, E. y Puig, L. (2014). Intertextuality and sense production in the learning of algebraic methods. Educational Studies in Mathematics, 87, 389-407. https://doi.org/10.1007/s10649-014-9561-3

ARMANDO SOLARES ROJAS

Dirección: Departamento de Matemática Educativa-Cinvestav IPN Av. Instituto Politécnico Nacional 2508, Col. San Pedro Zacatenco 07360 México, Ciudad de México.

Teléfonos: (52)+(55)-57-47-38-15 